\title{
Impact Analysis of Guidance Instrument Error Separation Accuracy on Sampling Rate of Inertial Navigation Equipment
}

\author{
Wen-jun Wang ${ }^{1 \mathrm{a}}$, Xiao-jun Duan ${ }^{1 \mathrm{~b}}$ and Ju-bo Zhu ${ }^{1 \mathrm{c}}$ \\ ${ }^{1}$ Department of Mathematics and System, Science College, National University of Defense \\ Technology, Changsha, 410073, China \\ accammer@tom.com, biaojun_duan@163.com, cjubo_zhu@163.com
}

Keywords: guidance instrument error separation; inertial navigation equipment; environmental function; data sampling rate; linear separation model; separation accuracy

\begin{abstract}
Based on the linear model of guidance instrument error separation, study on the separation accuracy affected by data sampling rate of inertial navigation equipment. First, theoretically proved that the higher data sampling rate is, the higher separation accuracy we can get. Second, a method for determining the optimal sampling rate is presented, whose idea is from the model itself. At last, the simulation results can verify the above two conclusions.
\end{abstract}

\section{Introduction}

Guidance instrument error separation is an important method for evaluating inertial navigation equipment performance and verifying the conformity between land tests and air tests. By evaluating the coefficient of guidance instrument error term, we can amend the inertial trajectory with the help of environmental function ${ }^{[1]}$. Zhang Z.L. gave the general idea of guidance instrument error separation first in china ${ }^{[2]}$, and he pointed out that the most basic methods of analysis is the standard integral method or the environment function method. Sha Y. et al transformed the problem of guidance instrument error separation into a linear regression problem ${ }^{[3]}$. Towards the linear regression problem, Luo \& Wu evaluated the estimation accuracy based on hypothesis testing method ${ }^{[4]}$.

According to the basic theory of point-estimation, it is right that "more sample, higher accuracy" ${ }^{\text {[5] }}$. But in problem of guidance instrument error separation, the target to be estimated is a vector. It seems that more samples will make better estimation accuracy ${ }^{[6]}$. But this conclusion is lack of rigorous theoretical proof. In another hand, it can not always be better when the sampling rate goes higher. There are two reasons: 1) it is high cost in hardware when the sampling rate goes higher; 2) usually it doesn't need very high sampling rate to meet the accuracy requirements. In summary, there must be an optimal sampling rate, and we can increase sampling rate until the estimation accuracy is enough towards the linear model and measured data.

Till now, the research on the relationship between data sampling rate and separation accuracy has not been reported in publications, which the paper talks about. In part 2, we theoretically proved that the relationship between the vector estimation accuracy and data sampling rate. In part 3 , a method for determining the best optimal sampling rate is presented based on the linear model. In part 4 , the theoretical results are verified by computing measured data.

\section{Linear separation model and separation accuracy analysis}

\section{Linear separation model}

Consider the linear model guidance instrument error separation in the velocity field ${ }^{[7-8]}$ :

$$
\mathbf{W}_{\mathbf{Y}}(t)-\mathbf{W}(t)=\mathbf{S}(\mathbf{W}(t), \dot{\mathbf{W}}(t)) \mathbf{C}+\mathbf{e}(t)
$$

Where $\mathbf{W}_{\mathbf{Y}}(t) \in \mathbb{R}^{n \times 1}$ stands for velocity vector data measured by inertial navigation equipment; $\mathbf{W}(t) \in \mathbb{R}^{n \times 1}$ stands for the real velocity vector data in inertial reference frame; $\mathbf{S}(\mathbf{W}(t), \dot{\mathbf{W}}(t)) \in \mathbb{R}^{n \times m}$ stands for the environmental function matrix made of velocity vectors; $\mathbf{C} \in \mathbb{R}^{n \times 1}$ stands for the 
guidance instrument error coefficient vector to be estimated; $\mathbf{e}(t) \in \mathbb{R}^{n \times 1}$ stands for model error vector.

Usually, we don't know the real velocity vector data in inertial reference frame, as an alternative we can use $\mathbf{W}_{\mathbf{w}_{2} \mathrm{Y}}(t)$ which stands for data translated from data measured in emission reference frame. So equation (1) can be rewritten as:

$$
\mathbf{W}_{\mathbf{Y}}(t)-\mathbf{W}_{\mathbf{W} 2 \mathbf{Y}}(t)=\mathbf{S}\left(\mathbf{W}_{\mathbf{w} 2 \mathbf{Y}}(t), \dot{\mathbf{W}}_{\mathbf{W} 2 \mathbf{Y}}(t)\right) \mathbf{C}+\mathbf{e}^{\prime}(t)
$$

For simple:

$$
\Delta \mathbf{W}=\mathbf{S C}+\mathbf{e}^{\prime}
$$

Where, $\mathbf{e}^{\prime} \in \mathbb{R}^{n \times 1}$ stands for the error vector, which contains the model error $\mathbf{e}(t)$ and translate error brought by $\mathbf{W}_{\mathbf{w} 2 \mathbf{Y}}(t)$, and $\mathbf{e}^{\prime}$ approximately obey the Gaussian distribution, that is $\mathbf{e}^{\prime}(t) \sim N(0, \sigma \mathbf{I})$.

\section{Accuracy of the least squares solution}

The least squares solution of problem (3) is ${ }^{[9]}$ :

$$
\hat{\mathbf{C}}=\left[\mathbf{S}^{T} \mathbf{S}\right]^{-1} \mathbf{S}^{T} \Delta \mathbf{W}
$$

$\hat{\mathbf{C}}$ is the estimation of guidance instrument error coefficient vector by least square, and its variance can also represent the estimation accuracy or separation accuracy. That is

$$
\begin{aligned}
\operatorname{cov}(\hat{\mathbf{C}}) & =E\left(\widehat{\mathbf{C}}^{2}\right)-E(\widehat{\mathbf{C}})^{2} \\
& =E\left\{\left[\mathbf{S}^{T} \mathbf{S}\right]^{-1} \mathbf{S}^{T}\left(\mathbf{S} \widehat{\mathbf{C}}+\mathbf{e}^{\prime}\right)\left(\mathbf{S} \widehat{\mathbf{C}}+\mathbf{e}^{\prime}\right)^{T} \mathbf{S}\left[\mathbf{S}^{T} \mathbf{S}\right]^{-1}\right\}-E(\widehat{\mathbf{C}})^{2} \\
& =E\left\{\left[\mathbf{S}^{T} \mathbf{S}\right]^{-1} \mathbf{S}^{T} \mathbf{S} \widehat{\mathbf{C}} \widehat{\mathbf{C}}^{T} \mathbf{S}^{T} \mathbf{S}\left[\mathbf{S}^{T} \mathbf{S}\right]^{-1}\right\}+E\left\{\left[\mathbf{S}^{T} \mathbf{S}\right]^{-1} \mathbf{S}^{T} \mathbf{e}^{\prime}\left(\mathbf{e}^{\prime}\right)^{T} \mathbf{S}\left[\mathbf{S}^{T} \mathbf{S}\right]^{-1}\right\} \\
& =E\left\{\widehat{\mathbf{C}} \widehat{\mathbf{C}}^{T}\right\}+\sigma^{2}\left[\mathbf{S}^{T} \mathbf{S}\right]^{-1}-E(\widehat{\mathbf{C}})^{2} \\
& =\sigma^{2}\left[\mathbf{S}^{T} \mathbf{S}\right]^{-1}
\end{aligned}
$$

So the variance of $\hat{\mathbf{C}}$ is:

$$
\operatorname{var}\left(\hat{\mathbf{C}}_{i i}\right)=\sigma^{2}\left\{\left[\mathbf{S}^{T} \mathbf{S}\right]^{-1}\right\}_{i i}
$$

Where subscript ii stands for the i th diagonal element of matrix. When we talk about the whole estimation accuracy, usually we refer to the trace of $\left[\mathbf{S}^{T} \mathbf{S}\right]^{-1}$. That is:

$$
\operatorname{Tr}\left(\left[\mathbf{S}^{T} \mathbf{S}\right]^{-1}\right)=\sum_{i=1}^{n} \operatorname{var}\left(\widehat{\mathbf{C}}_{i i}\right)
$$

The trace of $\left[\mathbf{S}^{T} \mathbf{S}\right]^{-1}$ is an important indicator of the estimation accuracy of guidance instrument error coefficient vector, and also is the object this paper focus on.

\section{Impact analysis of data sampling rate}

Consider the linear model of guidance instrument error separation: $\Delta \mathbf{W}=\mathbf{S C}+\mathbf{e}^{\prime}$, with $\mathrm{n}$ by $\mathrm{m}$ environmental function matrix $\mathbf{S}$, and usually $n>m$. Denote the matrix composed by added sample data by $\mathbf{S}_{b}$, so the augmented environment function matrix can be denoted as $\mathbf{G} \triangleq\left[\mathbf{S}, \mathbf{S}_{b}\right]^{T}$.

So the linear model can be rewritten as:

$$
\Delta \mathbf{W}^{\prime}=\mathbf{G C}+\mathbf{e}^{\prime}
$$

So that the accuracy index is changed into $\operatorname{Tr}\left[\mathbf{G}^{T} \mathbf{G}\right]^{-1}$ from $\operatorname{Tr}\left[\mathbf{S}^{T} \mathbf{S}\right]^{-1}$. Further, 


$$
\mathbf{G}^{T} \mathbf{G}=\left[\begin{array}{ll}
\mathbf{S}^{T} & \mathbf{S}_{b}^{T}
\end{array}\right]\left[\begin{array}{l}
\mathbf{S} \\
\mathbf{S}_{b}
\end{array}\right]=\mathbf{S}^{T} \mathbf{S}+\mathbf{S}_{b}^{T} \mathbf{S}_{b}
$$

Therefore

$$
\left[\mathbf{G}^{T} \mathbf{G}\right]^{-1}=\left[\mathbf{S}^{T} \mathbf{S}+\mathbf{S}_{b}^{T} \mathbf{S}_{b}\right]^{-1}
$$

According to the matrix seeking the inverse theorem ${ }^{[10]}$ :

$$
\begin{aligned}
{\left[\mathbf{G}^{T} \mathbf{G}\right]^{-1} } & =\left[\mathbf{S}^{T} \mathbf{S}+\mathbf{S}_{b}{ }^{T} \mathbf{S}_{b}\right]^{-1} \\
& =\left[\mathbf{S}^{T} \mathbf{S}\right]^{-1}-\left[\mathbf{S}^{T} \mathbf{S}\right]^{-1} \mathbf{S}_{b}^{T}\left(I+\mathbf{S}_{b}\left[\mathbf{S}^{T} \mathbf{S}\right]^{-1} \mathbf{S}_{b}^{T}\right)^{-1} \mathbf{S}_{b}\left[\mathbf{S}^{T} \mathbf{S}\right]^{-1}
\end{aligned}
$$

Next, what we should do is to prove the following formula :

$$
\operatorname{Tr}\left[\mathbf{G}^{T} \mathbf{G}\right]^{-1}<\operatorname{Tr}\left[\mathbf{S}^{T} \mathbf{S}\right]^{-1}
$$

First, without proof give five theorems about trace of matrix ${ }^{[10]}$ :

Theorem 1: If matrix $\mathbf{A}$ is positive and reversible, then its inverse matrix $\mathbf{A}^{-1}$ is also positive.

Theorem 2: $\operatorname{tr}(\mathbf{A}+\mathbf{B})=\operatorname{tr}(\mathbf{A})+\operatorname{tr}(\mathbf{B})$.

Theorem 3: $\operatorname{tr}(\mathbf{A B})=\operatorname{tr}(\mathbf{B A})$.

Theorem 4: If matrix $\mathbf{M}$ and matrix $\mathbf{N}$ are positive, then matrix $\mathbf{M N M}$ is positive too; if matrix $\mathbf{N}$ is positive semidefinite, then matrix $\mathrm{MNM}$ is positive semidefinite.

Theorem 5: If $\mathbf{x}$ is a $1 \times m$ vector, then $\mathbf{x}^{T} \mathbf{x}$ is positive semidefinite matrix, and it has only one eigenvalue $\lambda$ s.t. $\lambda=\mathbf{x x}^{T}$.

Proposition 1:

If matrix $\mathbf{S}$ satisfy $\operatorname{Rank}(\mathbf{S})=m$, and $\mathbf{S}_{b} \neq 0$, then $\operatorname{Tr}\left[\mathbf{G}^{T} \mathbf{G}\right]^{-1}>\operatorname{Tr}\left[\mathbf{S}^{T} \mathbf{S}\right]^{-1}$.

\section{Proof:}

(1) Because of $\operatorname{Rank}(\mathbf{S})=m$, so matrix $\mathbf{S}$ is column full rank. And we can get the equation $\operatorname{Rank}\left(\mathbf{S}^{T} \mathbf{S}\right)=m$. Do SVD (SVD: Singular Value Decomposition) on $\mathbf{S}$, that is $\mathbf{S}=\mathbf{U} \Sigma_{\mathbf{s}} \mathbf{V}^{H}$, where $\mathbf{U}$ and $\mathbf{V}$ are unitary matrix; and $\Sigma_{\mathbf{s}}$ satisfy the equation $\Sigma_{\mathbf{s}}=\left[\begin{array}{c}\operatorname{diag}\left(\gamma_{s i}\right)_{m \times m} \\ \mathbf{0}_{(n-m) \times m}\end{array}\right]_{n \times m}, \quad i=1,2, \ldots, m$. Then $\mathbf{S}^{T} \mathbf{S}=\mathbf{V} \operatorname{diag}\left(\gamma_{s i}^{2}\right) \mathbf{V}^{H}, \quad i=1,2, \ldots, m$, that is to say $\gamma_{s i}^{2}>0$ is the $i$ th eigenvalue of matrix $\mathbf{S}^{T} \mathbf{S}$, so we can make the conclusion: matrix $\mathbf{S}^{T} \mathbf{S}$ is positive definite, and so does $\left[\mathbf{S}^{T} \mathbf{S}\right]^{-1}$ according to Theorem 1.

(2) Consider a special situation when $\mathbf{S}_{1}$ is a $1 \times m$ matrix (vector), that is increasing one sample into environmental function matrix. Notice that $\left[\mathbf{S}^{T} \mathbf{S}\right]^{-1}$ is positive definite, so $\forall \mathbf{S}_{b} \neq 0$, the formula $\mathbf{S}_{1}\left[\mathbf{S}^{T} \mathbf{S}\right]^{-1} \mathbf{S}_{1}^{T}>0$ is always right according to Theorem 4.

Let $c \triangleq \mathbf{S}_{1}\left[\mathbf{S}^{T} \mathbf{S}\right]^{-1} \mathbf{S}_{1}^{T}$ be a constant, and $\left(I+\mathbf{S}_{1}\left[\mathbf{S}^{T} \mathbf{S}\right]^{-1} \mathbf{S}_{1}^{T}\right)^{-1}=\frac{1}{c+1}$. According to Theorem 2, we can get this equation:

$$
\operatorname{tr}\left[\mathbf{G}_{1}{ }^{T} \mathbf{G}_{1}\right]^{-1}=\operatorname{tr}\left[\mathbf{S}^{T} \mathbf{S}\right]^{-1}-\frac{1}{c+1} \operatorname{tr}\left\{\left[\mathbf{S}^{T} \mathbf{S}\right]^{-1} \mathbf{S}_{1}^{T} \mathbf{S}_{1}\left[\mathbf{S}^{T} \mathbf{S}\right]^{-1}\right\}
$$

Where $\mathbf{G}_{1} \triangleq\left[\mathbf{S}, \mathbf{S}_{1}\right]^{T}$.

(3) According to Theorem 3, 


$$
\begin{aligned}
\operatorname{tr}\left\{\left[\mathbf{S}^{T} \mathbf{S}\right]^{-1} \mathbf{S}_{1}{ }^{T} \mathbf{S}_{1}\left[\mathbf{S}^{T} \mathbf{S}\right]^{-1}\right\} & =\operatorname{tr}\left\{\mathbf{S}_{1}\left[\mathbf{S}^{T} \mathbf{S}\right]^{-1}\left[\mathbf{S}^{T} \mathbf{S}\right]^{-1} \mathbf{S}_{1}{ }^{T}\right\} \\
& =\operatorname{tr}\left\{\mathbf{S}_{1}\left[\mathbf{S}^{T} \mathbf{S}\right]^{-2} \mathbf{S}_{1}^{T}\right\} \\
& =\mathbf{S}_{1}\left[\mathbf{S}^{T} \mathbf{S}^{-2} \mathbf{S}_{1}^{T}\right.
\end{aligned}
$$

Again according to Theorem $4,\left[\mathbf{S}^{T} \mathbf{S}\right]^{-2}$ is positive definite too. So $\mathbf{S}_{1}\left[\mathbf{S}^{T} \mathbf{S}\right]^{-2} \mathbf{S}_{1}^{T}>0$

Then we can get:

$$
\begin{aligned}
& \operatorname{tr}\left[\mathbf{G}_{1}{ }^{T} \mathbf{G}_{1}\right]^{-1}=\operatorname{tr}\left[\mathbf{S}^{T} \mathbf{S}\right]^{-1}-\frac{1}{c+1} \operatorname{tr}\left\{\left[\mathbf{S}^{T} \mathbf{S}\right]^{-1} \mathbf{S}_{1}{ }^{T} \mathbf{S}_{1}\left[\mathbf{S}^{T} \mathbf{S}\right]^{-1}\right\} \\
& \because \frac{\mathbf{S}_{1}\left[\mathbf{S}^{T} \mathbf{S}\right]^{-2} \mathbf{S}_{1}^{T}}{c+1}>0, \therefore \operatorname{tr}\left[\mathbf{G}_{1}{ }^{T} \mathbf{G}_{1}\right]^{-1}<\operatorname{tr}\left[\mathbf{S}^{T} \mathbf{S}\right]^{-1} .
\end{aligned}
$$

(4) Now, we will prove Proposition1 by mathematical induction method.

Suppose $\mathbf{S}_{b}$ is a $b \times m$ matrix, $\mathbf{G}_{b} \triangleq\left[\mathbf{S}, \mathbf{S}_{b}\right]^{T}$, so $\mathbf{G}_{b+1} \triangleq\left[\mathbf{S}_{1}, \mathbf{G}_{b}\right]^{T}$, according to (2) and (3), $\operatorname{tr}\left[\mathbf{G}_{b+1}{ }^{T} \mathbf{G}_{b+1}\right]^{-1}>\operatorname{tr}\left[\mathbf{G}_{b}^{T} \mathbf{G}_{b}\right]^{-1}$ is right. So we can get this equation:

$$
\operatorname{tr}\left[\mathbf{G}_{b+1}{ }^{T} \mathbf{G}_{b+1}\right]^{-1}>\operatorname{tr}\left[\mathbf{G}_{b}^{T} \mathbf{G}_{b}\right]^{-1}>\ldots>\operatorname{tr}\left[\mathbf{G}_{1}^{T} \mathbf{G}_{1}\right]^{-1}>\operatorname{tr}\left[\mathbf{S}^{T} \mathbf{S}\right]^{-1}
$$

According to mathematical induction method, when $\mathbf{S}_{k}$ is a $k \times m$ matrix, $k \geq 1$, and $\mathbf{S}_{k} \neq 0$, we can get $\operatorname{tr}\left[\mathbf{G}^{T} \mathbf{G}\right]^{-1}<\operatorname{tr}\left[\mathbf{S}^{T} \mathbf{S}\right]^{-1}$.

\section{Proposition 2:}

Especially, when $\mathbf{S}_{b} \doteq \mathbf{S}$, which means when the sampling time interval of original environmental function matrix $\mathbf{S}$ is short enough, and $\mathbf{S}_{b}$ is generated by the mid-point interpolation method, then we can consider that $\mathbf{S}_{b}$ approximately equals to $\mathbf{S}$, then the equation $\operatorname{tr}\left[\mathbf{G}^{T} \mathbf{G}\right]^{-1} \doteq \frac{1}{2} \operatorname{tr}\left[\mathbf{S}^{T} \mathbf{S}\right]^{-1}$ is approximately right.

\section{Proof:}

Because of $\mathbf{S}^{T} \mathbf{S}=\operatorname{Vdiag}\left(\gamma_{s i}^{2}\right) V^{H}$, so $\left[\mathbf{S}^{T} \mathbf{S}\right]^{-1}=\mathbf{V} \operatorname{diag}\left(\gamma_{i}^{-2}\right) \mathbf{V}^{H}$.

Do SVD on $\mathbf{S}_{b}$, we get $\mathbf{S}_{b}=\mathbf{U}_{b} \Sigma_{b} \mathbf{V}_{b}^{H}$, where $\mathbf{U}_{b}$ and $\mathbf{V}_{b}$ are unitary matrix; and $\Sigma_{b}$ satisfy the equation $\Sigma_{b}=\left[\begin{array}{c}\operatorname{diag}\left(\gamma_{b i}\right)_{m \times m} \\ \mathbf{0}_{(n-m) \times m}\end{array}\right]_{n \times m}, i=1,2, \ldots, m$.

$$
\begin{aligned}
& \because \mathbf{S}_{b} \doteq \mathbf{S}, \therefore \mathbf{U}_{b} \doteq \mathbf{U}, \quad \mathbf{V}_{b} \doteq \mathbf{V}, \Sigma_{b} \doteq \Sigma_{s} \text {, what is more we can get } \\
& \mathbf{S}_{b}\left[\mathbf{S}^{T} \mathbf{S}\right]^{-1} \mathbf{S}_{b}^{T}=\mathbf{U}_{b} \Sigma_{b} \mathbf{V}_{b}^{H} \mathbf{V} \Sigma_{s}{ }^{-2} \mathbf{V}^{H} \mathbf{V}_{b} \Sigma_{b} \mathbf{U}_{b}^{H} \doteq I_{k}
\end{aligned}
$$

Then

$$
\begin{aligned}
& \operatorname{tr}\left\{\left[\mathbf{S}^{T} \mathbf{S}\right]^{-1} \mathbf{S}_{b}^{T}\left(I+\mathbf{S}_{b}\left[\mathbf{S}^{T} \mathbf{S}\right]^{-1} \mathbf{S}_{b}{ }^{T}\right)^{-1} \mathbf{S}_{b}\left[\mathbf{S}^{T} \mathbf{S}\right]^{-1}\right\} \\
= & \operatorname{tr}\left\{\mathbf{S}_{b}\left[\mathbf{S}^{T} \mathbf{S}\right]^{-1}\left[\mathbf{S}^{T} \mathbf{S}\right]^{-1} \mathbf{S}_{b}{ }^{T}\left(I_{k}+I_{k}\right)^{-1}\right\} \\
= & \frac{1}{2} \operatorname{tr}\left\{\mathbf{S}_{b}\left[\mathbf{S}^{T} \mathbf{S}\right]^{-1}\left[\mathbf{S}^{T} \mathbf{S}\right]^{-1} \mathbf{S}_{b}{ }^{T}\right\} \\
= & \frac{1}{2} \operatorname{tr}\left\{\mathbf{S}_{b}{ }^{T} \mathbf{S}_{b}\left[\mathbf{S}^{T} \mathbf{S}\right]^{-1}\left[\mathbf{S}^{T} \mathbf{S}\right]^{-1}\right\} \\
\doteq & \frac{1}{2} \operatorname{tr}\left\{\left[\mathbf{S}^{T} \mathbf{S}\right]^{-1}\right\}
\end{aligned}
$$

\section{Proposition 3:}


When the sampling time interval of original environmental function matrix $\mathbf{S}$ is short enough, and do $l$ times of mid-point interpolation on $\mathbf{S}$, so that the sample number of $\mathbf{G}$ is $2^{l}$ times of $\mathbf{S}$, then $\operatorname{tr}\left[\mathbf{G}^{T} \mathbf{G}\right]^{-1} \doteq \frac{1}{2^{l}} \operatorname{tr}\left\{\left[\mathbf{S}^{T} \mathbf{S}\right]^{-1}\right\}$.

Proof:

According to the result of Proposition 2, Proposition 3 is obviously right.

\section{Constraints on sampling data from trajectory accuracy}

In fact, there should be a reasonable sup of data sampling rate. That is to say, it doesn't need a very high data sampling rate to achieve the accuracy requirement in trajectory calculation. Consider linear model $\Delta \mathbf{W}=\mathbf{S C}+\mathbf{e}^{\prime}$, we consider $\mathbf{S C}$ as the system component of $\Delta \mathbf{W}$, and $\mathbf{e}^{\prime}$ as the random component. So, when we get the estimation $\hat{\mathbf{C}}$, the estimation of system component of $\Delta \mathbf{W}$ is $\mathbf{S} \hat{\mathbf{C}}$.

Since $\hat{\mathbf{C}}$ is estimation value, so it has estimation error. We can believe that $\hat{\mathbf{C}}$ obeys the normal distribution, as the follow equation shows:

$$
\begin{aligned}
& \hat{\mathbf{C}}_{i} \sim N\left(\mathbf{C}_{i}, \sigma_{i}\right) \\
& \sigma_{i} \triangleq \sqrt{\operatorname{var}\left(\widehat{\mathbf{C}}_{i i}\right)}=\sigma \sqrt{\left\{\left[\mathbf{S}^{T} \mathbf{S}\right]^{-1}\right\}_{i i}}, i=1,2, \ldots, m, \\
& \mathbf{S} \hat{\mathbf{C}} \sim N(\mathbf{S C}, \mathbf{S} \Delta), \Delta \triangleq\left(\sigma_{1}, \sigma_{2}, \ldots, \sigma_{m}\right)^{T}
\end{aligned}
$$

In order to guarantee that the system component of $\Delta \mathbf{W}$ is fully extracted, the magnitude of $\mathrm{S} \Delta$ should be much more less than magnitude of random component, that is:

$$
\max (\mathbf{S} \Delta) \ll \max \left(\Delta_{\mathbf{e}^{\prime}}\right)
$$

Since the variance of random component $\sigma$ is regarded as a constant, so (19) can be rewritten as:

$\max (\mathbf{S} \Delta) \ll \sigma$

We call $\max (\mathbf{S} \Delta)$ separation accuracy characteristic value. Take (18) into (21), that is:

$$
\max _{i}\left(\mathbf{S} \sigma v e c\left\{\sqrt{\left[\mathbf{S}^{T} \mathbf{S}\right]^{-1}}\right\}\right) \ll \sigma
$$

Where, vec $\left\{\sqrt{\left[\mathbf{S}^{T} \mathbf{S}^{-1}\right.}\right\} \triangleq\left\{\sqrt{\left[\mathbf{S}^{T} \mathbf{S}\right]^{-1}}, \sqrt{\left[\mathbf{S}^{T} \mathbf{S}\right]^{-1}}, \ldots, \sqrt{\left[\mathbf{S}^{T} \mathbf{S}\right]^{-1}{ }_{m m}}\right\}^{T}$, then delete $\sigma$ together:

$$
\max _{i}\left(\mathbf{S} v e c\left\{\sqrt{\left[\mathbf{S}^{T} \mathbf{S}^{-1}\right.}{ }_{i i}\right\}\right) \ll 1
$$

As the increase of sampling rate, $\sqrt{\left[\mathbf{S}^{T} \mathbf{S}\right]^{-1}}$ and $\max _{i}\left(\mathbf{S} v e c\left\{\sqrt{\left[\mathbf{S}^{T} \mathbf{S}\right]^{-1}}\right\}\right)$ both will be diminished. When $\max _{i}\left(\mathbf{S} v e c\left\{\sqrt{\left[\mathbf{S}^{T} \mathbf{S}\right]^{-1}}\right\}\right)$ is lower than 0.01, we consider the sampling rate as the optimal data sampling rate.

\section{Simulation}

\section{Verification of direct proportion between sampling rate and estimation accuracy}

This section verifies the conclusions of part 2. Base on the measurement of position and speed in the whole trajectory, we can get data in various sampling rate, such as $10 \mathrm{~Hz}, 20 \mathrm{~Hz}, 40 \mathrm{~Hz}$ and $80 \mathrm{~Hz}$. Compute the value of $\operatorname{tr}\left[\mathbf{S}^{T} \mathbf{S}\right]^{-1}$, record the result in table 1:

Table 1 value of $\operatorname{tr}\left[\mathbf{S}^{T} \mathbf{S}\right]^{-1}$ in different sampling rates 


\begin{tabular}{|c|c|c|c|c|}
\hline Data sampling rate & $10 \mathrm{~Hz}$ & $20 \mathrm{~Hz}$ & $40 \mathrm{~Hz}$ & $80 \mathrm{~Hz}$ \\
\hline $\operatorname{tr}\left[\mathbf{S}^{T} \mathbf{S}^{-1}\right.$ & $3.42 \mathrm{e} 10$ & $1.72 \mathrm{e} 10$ & $8.63 \mathrm{e} 9$ & $4.32 \mathrm{e} 9$ \\
\hline
\end{tabular}

Form the computing result, we can see that, as the data sampling rate has been double heightened, the value of $\operatorname{tr}\left[\mathbf{S}^{T} \mathbf{S}\right]^{-1}$ reduced in the same proportion, which can verify the conclusion of Proposition 3. So we can believe the general conclusion: “The higher data sampling rate, the higher the estimation accuracy of guidance instrument error separation."

\section{Verification of separation accuracy characteristic value}

Use the same method as section 4.1 , we can get data of $10 \mathrm{~Hz}, 20 \mathrm{~Hz}, 40 \mathrm{~Hz}$ and $80 \mathrm{~Hz}$. Compute the values of $\max _{i}\left(\mathbf{S} v e c\left\{\sqrt{\left[\mathbf{S}^{T} \mathbf{S}\right]^{-1}}\right\}\right)$, and draw the result in fig.1.

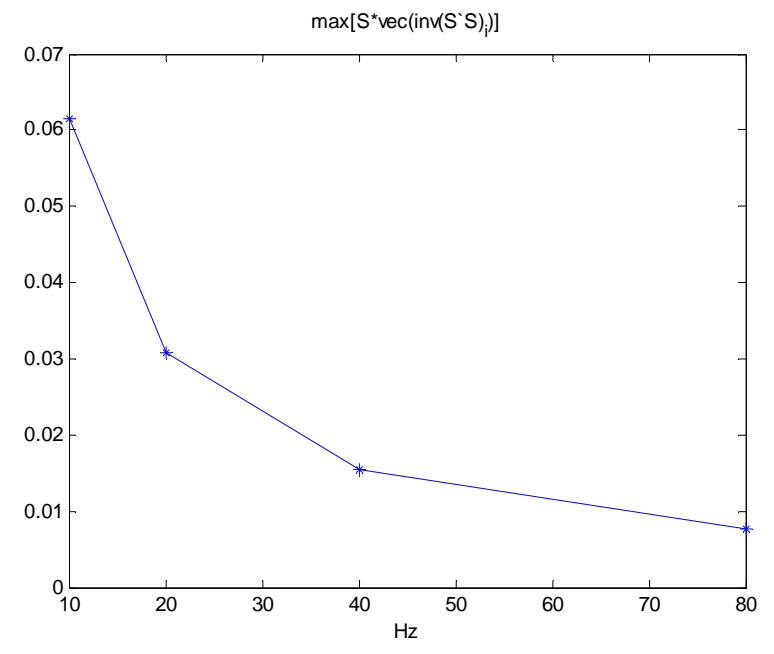

Figure 1 values of $\max _{i}\left(\mathbf{S} v e c\left\{\sqrt{\left[\mathbf{S}^{T} \mathbf{S}\right]^{-1}}\right\}\right)$ in different sampling rates

From fig.1, we can see when the sampling rate reaches $60 \mathrm{~Hz}$, the separation accuracy characteristic value is close to 0.01 , which nearly meet the conditions of equation (23). So, for this measured data, the proper data sampling rate should be $60 \mathrm{~Hz}$.

\section{Conclusion}

First, theoretically deduced the relationship between data sampling rate and separation accuracy. Then, a method for determining an optimal sampling rate based on the linear model is given. At last, we verified our conclusions by measured data computing.

Generally speaking, enhancing data sampling rate will get higher estimation accuracy in guidance instrument error separation. But, when the sampling rate is above a certain value, it is not meaningful to continue improvement. According the method presented in this article, we can determine a proper or an optimal data sampling rate for any measured data. Further, by lots of data computing of the same inertial navigation equipment, we can get the best sampling rate of the equipment. In summary, the method presented can be use in experiment design and equipment hardware design.

\section{Acknowledgments}

First, I should give my best regard to Professor Ju-bo Zhu who usually tell me a big success always exist in a little problem. Also I appreciate all the teachers in the department of mathematics and systems science and the members in group 4202, who gave us many useful suggestions. Thank you! 
This work is supported by the Natural Science Foundation of China (60974124), the Program for New Century Excellent Talents in University and the Project-sponsored by SRF for ROCS, SEM in China, the key lab open foundation for Space Flight Dynamics technique (SFDLXZ-2010-004).

\section{References}

[1] Jia Pei-ran, Use Special Trajectory Error Coefficient of Guidance Tool and Deduce Guidance Tool Error of Normal Trajectory [J]. Journal of National University of Defense Technology, 1985, (02): 59-66.

[2] Zhang Zui-liang, A Notation on the Analysis of the Error in Inertial Guidance Tools [J]. Journal of National University of Defense Technology, 1981, (04): 83 88.

[3] Sha Yu, Wu Yi, Wang Zheng-ming, Development and Improvement of Principal Components Estimation and Its Application in the Separation of Guidance Instrumental Tool Errors[J]. Aerospace Control, 1991, (S1): 136-146.

[4] Luo Jian-shu, Wu Yi, The Method for Appraising Accuracy of Estimated Values of Regression Coefficients by Hypothesis Test[J]. Missile and Space Vehicles, 1994, (01): 23-29.

[5] Wang Zheng-ming, Yi Dong-yun, The Calibration and Evaluation of Trajectory Tracking Data. Changsha: Publishing House of National University of Defence Technology, 1999, pp328-384.

[6] Cheng Guang-xian, Selection of Data Reasonableness Check and Error Separation Methods[J]. Aerospace Control, 1991, (S1): 104-111.

[7] Xu Ai-ping, An Instrumental Error Analysis Method for Strap-down Guidance System[J]. Aerospace Control, 1992, (02): 34-39.

[8] Zhao Min, Conversion Method of Guidance Instrumental Error[J]. Missile and Space Vehicles, 1993, (01): 42-48.

[9] Yao Jing, Duan Xiao-jun, Zhou Hai-yin, Modeling and Parameters Estimation of Marine Guidance Instrumental Systematic Error[J]. Journal of Ballistics, 2005， (01): : 33-39.

[10] Zhang Xian-da, Matrix Analysis and Applications[D], Springer, 2004: 54-56. 